\title{
Molecular Interaction between Projection Neuron Precursors and Invading Interneurons via Stromal-Derived Factor 1 (CXCL12)/CXCR4 Signaling in the Cortical Subventricular Zone/Intermediate Zone
}

\author{
Marie-Catherine Tiveron, ${ }^{1}$ Mireille Rossel, ${ }^{2}$ Barbara Moepps, ${ }^{3}$ Yong Li Zhang, ${ }^{1}$ Ralph Seidenfaden, ${ }^{1}$ Jack Favor, ${ }^{4}$ \\ Norbert König, ${ }^{2}$ and Harold Cremer ${ }^{1}$ \\ ${ }^{1}$ Institut de Biologie du Développement de Marseille Luminy, Centre National de la Recherche Scientifique/Université de Mediterranée, 13288 Marseille, \\ France, ${ }^{2}$ EPHE, Biologie Cellulaire Quantitative, Université Montpellier 2, Institut National de la Santé et de la Recherche Médicale (INSERM), Equipe Mixte \\ INSERM 343, 34095 Montpellier, France, ${ }^{3}$ Department of Pharmacology and Toxicology, University of Ulm, 89081 Ulm, Germany, and ${ }^{4}$ Gesellschaft für \\ Strahlung und Umweltforschung, 85764 Neuherberg, Germany
}

\begin{abstract}
Most cortical interneurons are generated in the subpallial ganglionic eminences and migrate tangentially to their final destinations in the neocortex. Within the cortex, interneurons follow mainly stereotype routes in the subventricular zone/intermediate zone (SVZ/IZ) and in the marginal zone. It has been suggested that interactions between invading interneurons and locally generated projection neurons are implicated in the temporal and spatial regulation of the invasion process. However, so far experimental evidence for such interactions is lacking.

We show here that the chemokine stromal-derived factor 1 (SDF-1; CXCL12) is expressed in the main invasion route for cortical interneurons in the SVZ/IZ. Most SDF-1-positive cells are proliferating and express the homeodomain transcription factors Cux1 and Cux2. Using MASH-1 mutant mice in concert with the interneuron marker DLX, we exclude that interneurons themselves produce the chemokine in an autocrine manner. We conclude that the SDF-1-expressing cell population represents the precursors of projection neurons during their transition and amplification in the SVZ/IZ. Using mice lacking the SDF-1 receptor CXCR4 or Pax6, we demonstrate that SDF-1 expression in the cortical SVZ/IZ is essential for recognition of this pathway by interneurons. These results represent the first evidence for a molecular interaction between precursors of projection neurons and invading interneurons during corticogenesis.
\end{abstract}

Key words: neocortex; migration; tangential; Cux1; Cux2; pallium; subpallium

\section{Introduction}

Whereas glutamatergic projection neurons of the rodent neocortex are generated locally in the dorsal forebrain (or pallium), GABAergic interneurons are generated in the ganglionic eminences (GEs) of the ventral forebrain (or subpallium) and invade the cortex via tangential long-distance migration, thereby using well defined migration routes in the subventricular zone/intermediate zone (SVZ/IZ) and the marginal zone (MZ) (for review,

\footnotetext{
Received June 14, 2006; revised Nov. 7, 2006; accepted Nov. 9, 2006.

This work was supported by grants from the European Community Network of Excellence "NeuroNE" (LSHM-CT2004-512039), the Fondation pour la Recherche sur le Cerveau, the Association Française contre les Myopathies, and the Fondation de France. R.S. was supported by a fellowship from the Deutsche Forschungsgemeinschaft. We thank Dr. N. Abrous for help with statistical analysis and A. Desoeuvre for expert technical assistance. We are grateful to $F$ Guillemot for Mash1 mutant mice, to D. Littman for CXCR4 mutants, to V. Pachnis for the LhX6 probe, to G. Panganiban for the anti-DLX antibody, and to C. Goridis for advice on double in situ hybridization. We thank C. Goridis, R. Kelly, and P. Carroll for critical comments on this manuscript.

Correspondence should be addressed to either of the following: Dr. Harold Cremer, Developmental Biology Institute of Marseille Luminy, Campus de Luminy case 907, 13288 Marseille cedex 9, France, E-mail: cremer@ibdm.univ-mrs.fr; Dr. Norbert König, University Montpellier 2, (c 103, Place E. Bataillon, 34095 Montpellier cedex 5, France, E-mail: koenig@univ-montp2.fr.

DOI:10.1523/JNEUROSCI.4162-06.2006

Copyright $\odot 2006$ Society for Neuroscience $\quad$ 0270-6474/06/2613273-06\$15.00/0
}

see Marin and Rubenstein, 2003). During their journey, they encounter radially migrating projection neurons, and there is evidence that the birthdates of principal neurons and interneurons are partially correlated within a particular cortical layer (for review, see Metin et al., 2006). Thus, the process of interneuron invasion is spatially and temporally regulated, suggesting interactions between both cell populations.

Several factors have been identified as potential regulators of cortical interneuron migration. These include semaphorins, Neuregulin-1, and glial cell line-derived neurotrophic factor (for review, see Metin et al., 2006). Furthermore, it has been reported that stromal-derived factor 1 (SDF-1) is a chemoattractant for cortical interneurons that is expressed not in the cortex itself but exclusively by meningeal cells (Stumm et al., 2003). However, several groups have reported $S D F-1$-expressing cells in the deeper aspect of the embryonic neocortex (Tham et al., 2001; Tissir et al., 2004), and this expression was associated with invading prospective interneurons (Daniel et al., 2005).

In this work, we identify the deep expression domain of SDF-1 in the developing cortex as the SVZ/IZ and investigate how this major pathway for interneurons is defined. We demonstrate that 
SDF-1 transcripts in the SVZ/IZ colocalize widely with Cux1 and Cux2, transcription factors that identify upper-layer cortical neurons and their precursors (Nieto et al., 2004; Zimmer et al., 2004; for review, see Rash and Grove, 2006), and exclude that SDF-1 expression is an autocrine function of interneurons via the use of specific markers and Mash-1 mutant mice. Furthermore, we show through the analysis of CXCR4 and Pax 6 mutant mice that SDF-1 is necessary for guidance of interneurons in the SVZ/IZ pathway.

These results provide the first demonstration of a molecular interaction between cortical projection neurons and interneurons before these reach their final destinations in the cortical layers.

\section{Materials and Methods}

Animals. All animals were treated according to protocols approved by the French Ethical Committee. CD1 mice (Iffa-Credo, Lyon, France) were used for expression pattern analyses. CXCR4 (Zou et al., 1998), Mash1 (Guillemot et al., 1993), and $\mathrm{Pax}^{3 \mathrm{Neu}}$ (Favor et al., 2001) mutant mice were maintained on a C57BL/6J background.

In situ hybridization and immunohistochemistry. Antisense RNA probes for Cux2, Dlx5 (Zimmer et al., 2004), Lhx6 (Lavdas et al., 1999), and SDF-1 (Daniel et al., 2005) were generated using the Dig-RNA labeling kit (Roche, Welwyn Garden City, UK). Tissue preparation, in situ hybridization (ISH), and combined ISH with immunohistochemistry were described previously (Tiveron et al., 1996; Daniel et al., 2005). DllDLX polyclonal rabbit IgG (Panganiban et al., 1995) was used 1:100. To detect proliferating cell nuclear antigen (PCNA; MAB377, 1:100; Chemicon, Temecula, CA) and Cux1 [CDP (C-20), 1:100; Santa Cruz Biotechnology, Santa Cruz, CA), sections were pretreated in boiling $10 \mathrm{~mm}$ citric acid, pH 6, for $60 \mathrm{~s}$. For double fluorescent ISH (FISH), sections were cohybridized with a digoxigenin (DIG)-labeled SDF probe and an FITClabeled Cux2 probe or with a DIG-labeled CXCR4 probe and an FITClabeled $L h x 6$ probe and processed as described by Dufour et al. (2006).

Quantitative analysis. Combining phase-contrast imaging, 4',6diamidino-2-phenylindole (DAPI) staining, and confocal microscopy, we analyzed 880 Cux2/SDF-1 cells on seven images. Only cells for which the assignment of staining was doubtlessly possible were counted. Lhx6positive cells (see Fig. 4) [three embryonic day 14.5 (E14.5) embryos per genotype] were counted on at least three coronal sections. The cortex was equally divided into six bins positioned parallel to the brain surface. The sections analyzed at the level of the medial GE/lateral GE spanned 500 $\mu \mathrm{m}$. ANOVA, followed by the Newman-Keuls post hoc test, was used for statistical analysis of data. Error bars represent SD.

\section{Results}

\section{SDF-1 expression in the cortical SVZ/IZ}

ISH for SDF-1 at E14.5 demonstrated strong expression in the meninges and a region between the cortical plate $(\mathrm{CP})$ and the ventricular zone (VZ) (Fig. 1a). Comparison of SDF-1 expression to that of Cux2 (Nieto et al., 2004; Zimmer et al., 2004) suggested that the deep SDF-1 expression domain was contained within the SVZ/IZ (Fig. 1b).

We performed a comparative time course analysis of $S D F-1$ and Cux2 expression during corticogenesis. At E13.5, serial sections stained for SDF-1 (Fig. 1c) and Cux2 (Fig. 1d) suggested that, as at E14.5, the SDF-1 domain in the SVZ/IZ (Fig. 1c) is overlapping with the $\mathrm{Cu} \times 2^{+}$domain (Fig. 1d). At E15.5, the SVZ/IZ domain contained large amounts of $\mathrm{Cu} \times 2^{+}$cells. Individual $\mathrm{Cu} \times 2^{+}$cells showing a radial orientation were visible in the already formed deep layers of the CP, and Cux2 ${ }^{+}$upper-layer neurons started accumulating in the CP (Fig. $1 f$ ). At this stage, $S D F-1$ expression was still strong in the meninges (Fig. 1e), whereas it was weaker and limited mainly to the deep aspect in the SVZ/IZ. At E18.5, the majority of Cux $2^{+}$upper-layer neurons had shifted to the CP, whereas the SVZ/IZ pool was strongly

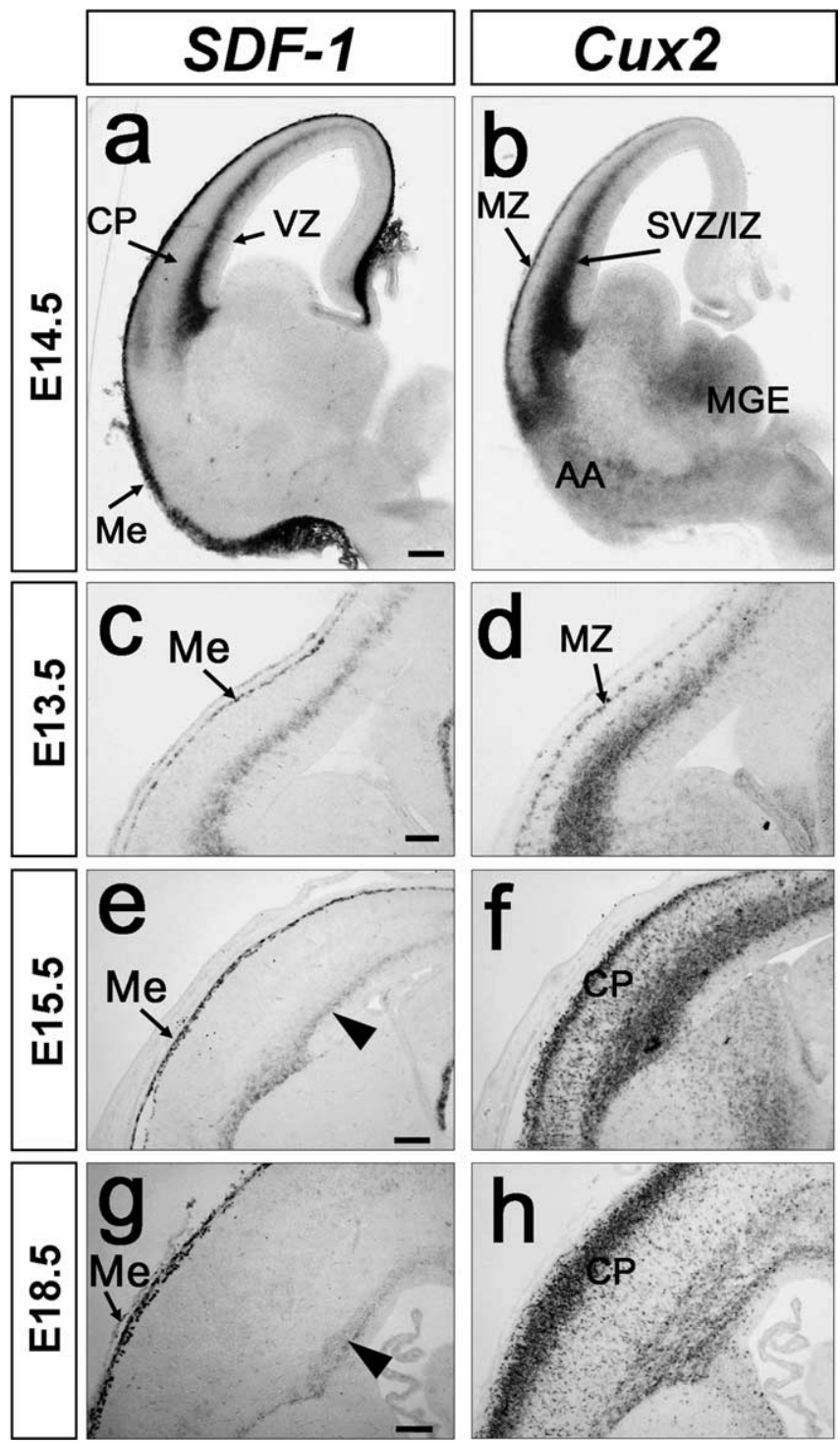

Figure 1. Expression of SDF-1 and Cux2 in the developing forebrain. ISH for SDF-1 $(\boldsymbol{a}, \boldsymbol{c}, \boldsymbol{e}, \boldsymbol{g})$ and $\operatorname{Cux2}(\boldsymbol{b}, \boldsymbol{d}, \boldsymbol{f}, \boldsymbol{h})$ on frontal serial forebrain vibratome sections at E14.5 $(\boldsymbol{a}, \boldsymbol{b})$ and cryostat sections at E13.5 (c, $\boldsymbol{d})$, E15.5 (e, $\boldsymbol{f})$, and E18.5 (g, $\boldsymbol{h}) . \boldsymbol{a}, \boldsymbol{c}$, At E13.5 and E14.5, SDF-1 expression is confined to the meninges and a cell population deep within the pallium. $\boldsymbol{b}, \boldsymbol{d}$, Cux2 labels the $\mathrm{SVZ} / \mathrm{IZ}$, interneurons in the $\mathrm{MZ}$, and the first cohort of upper-layer neurons. Cux2 staining extends into the ventral forebrain. The deep SDF-1 expression domain $(\boldsymbol{a}, \boldsymbol{c})$ is mainly overlapping with the Cux2 domain, demarcating the SVZ/IZ (b, d). e, $\boldsymbol{g}$, At E15.5 (e) and E18.5 (g), SDF-1 expression is strong in the meninges but is confined to the deep aspect in the SVZ/IZ (arrowhead). $\boldsymbol{f}, \boldsymbol{h}$, At the same time, Cux2 expression is found in upper-layer neurons of the developing CP. Furthermore, cells in the SVZ/IZ and radially oriented cells traversing the already formed deep layers express the transcription factor. Scale bars: $\boldsymbol{a}, \boldsymbol{b}, \boldsymbol{e}-\boldsymbol{h}, 200 \mu \mathrm{m} ; \boldsymbol{c}, \boldsymbol{d}, 100 \mu \mathrm{m}$. Me, Meninges; AA, amygdala anlage; MGE, medial GE.

diminished (Fig. 1h). At this age, SDF-1 expression in the SVZ/IZ was faint, whereas expression remained strong in the meninges (Fig. $1 g$ ).

Thus, SDF-1 expression in the embryonic pallium was confined to two major locations, the SVZ/IZ and the meninges. Expression over time was stable in the meninges. In the SVZ/IZ pathway, it was highly dynamic and partially overlapped with the expression of the upper-layer neuron marker Cux2.

\section{Projection neuron precursors express SDF-1}

We aimed at identifying the SDF-1-expressing cell populations in the SVZ/IZ. Recent studies have shown that precursors of CP 
neurons undergo cell divisions in the SVZ/IZ (Haubensak et al., 2004; Miyata et al., 2004; Noctor et al., 2004; Wu et al., 2005). The transcription factors Cux2 and Cux 1 are expressed in upper-layer neurons as well as their proliferating precursors in the SVZ/IZ (Nieto et al., 2004; Zimmer et al., 2004; Rash and Grove, 2006). Therefore, we asked whether the transcription factors were colocalized with SDF-1 at the cellular level.

In the absence of functional antibodies against $\mathrm{Cux} 2$ or SDF-1, we used FISH (Fig. 2a,b), allowing the detection of both transcripts on the same section. Low-magnification imaging verified the general overlap of Cux2 and SDF-1 expression at E13.5 mainly in the deep aspect of the SVZ/IZ, whereas more superficial areas were positive for Cux2 only. High-magnification confocal images showed the punctiform appearance of the respective fluorescent labels as separate signals within individual cells, because of the local precipitation of fluorescent substrate (Fig. $2 b$ ). Such high-magnification images in concert with phase-contrast imaging and DAPI nuclear staining allowed the identification of individual cells and their content of Cux2- and SDF-1-specific precipitates. Quantitative analyses revealed that $52.2 \pm 3.9 \%$ of all cells in the SVZ/IZ expressed both SDF-1 and Cux2. A total of $39.0 \pm 8.9 \%$ of the cells expressed only Cux2. These were mainly, but not exclusively, localized in the superficial aspect of the SVZ/ IZ. Finally, $8.8 \pm 4.9 \%$ expressed SDF-1 only (Fig. 2 c).

In the next step, we combined immunohistochemistry for Cux1 with ISH for SDF-1. At E15.5, Cux1 was strongly expressed in the developing upper layers of the $\mathrm{CP}$ and more weakly in the SVZ/IZ as well as in the VZ (Fig. 2d), whereas it was confined to individual radially oriented cells in the deep layers (Nieto et al., 2004). SDF-1 colocalized with Cux 1 in the SVZ/IZ but was absent from the strongly Cux1-positive cells in the upper cortical layers (Fig. $2 d, e$ ). Examination of 410 individual SDF-1-positive cells in the SVZ/IZ revealed that $94.9 \%$ coexpressed Cux1.

Thus, SDF-1 in the SVZ/IZ was mainly, but not exclusively, present in cells that coexpressed the transcription factors Cux2 and Cuxl, indicating that precursors of principal neurons are responsible for expression of the chemokine. Because such precursors have been shown to divide in the SVZ/IZ, we combined ISH for SDF-1 with immunolabeling for the cell-cycle marker PCNA (Fig. 2f) (Takahashi and Caviness, 1993). Analyses of 906 cells in the SVZ/IZ revealed that $68.9 \%$ of the SDF-1-positive cells in the SVZ/IZ were proliferating, in agreement with a projection neuron precursor identity of the SDF-1-expressing cell population.

Cux2 is expressed by precursors of principal neurons in the SVZ/IZ as well as by interneurons invading from the GE (Zimmer et al., 2004). Therefore, we asked whether SDF-1 expression is also a property of interneurons during their migration in the SVZ/IZ. We combined SDF-1 ISH with immunolabeling using a pan-DLX antibody. The complete absence of double-positive cells ( 10 sections from two embryos analyzed) (Fig. $2 g$ ) indicated SDF-1 expression in cells other than interneurons. We used a genetic model to further exclude a possible implication of interneurons in SDF-1 expression. Mash1 knock-out mice show a severe reduction in neuronal progenitors in the ventral telencephalon (Casarosa et al., 1999), leading to a loss of cortical interneurons, as identified by $D l x 5$ expression, in the MZ as well as in the SVZ/IZ (Fig. 2i,k). However, SDF-1 expression was not apparently reduced in the SVZ/IZ of E13.5 Mash1 mutants (Fig. $2 h, j)$.

Altogether, the findings that the majority of SDF- 1 cells in the SVZ/IZ (1) expressed the transcription factors Cux1 and Cux2,
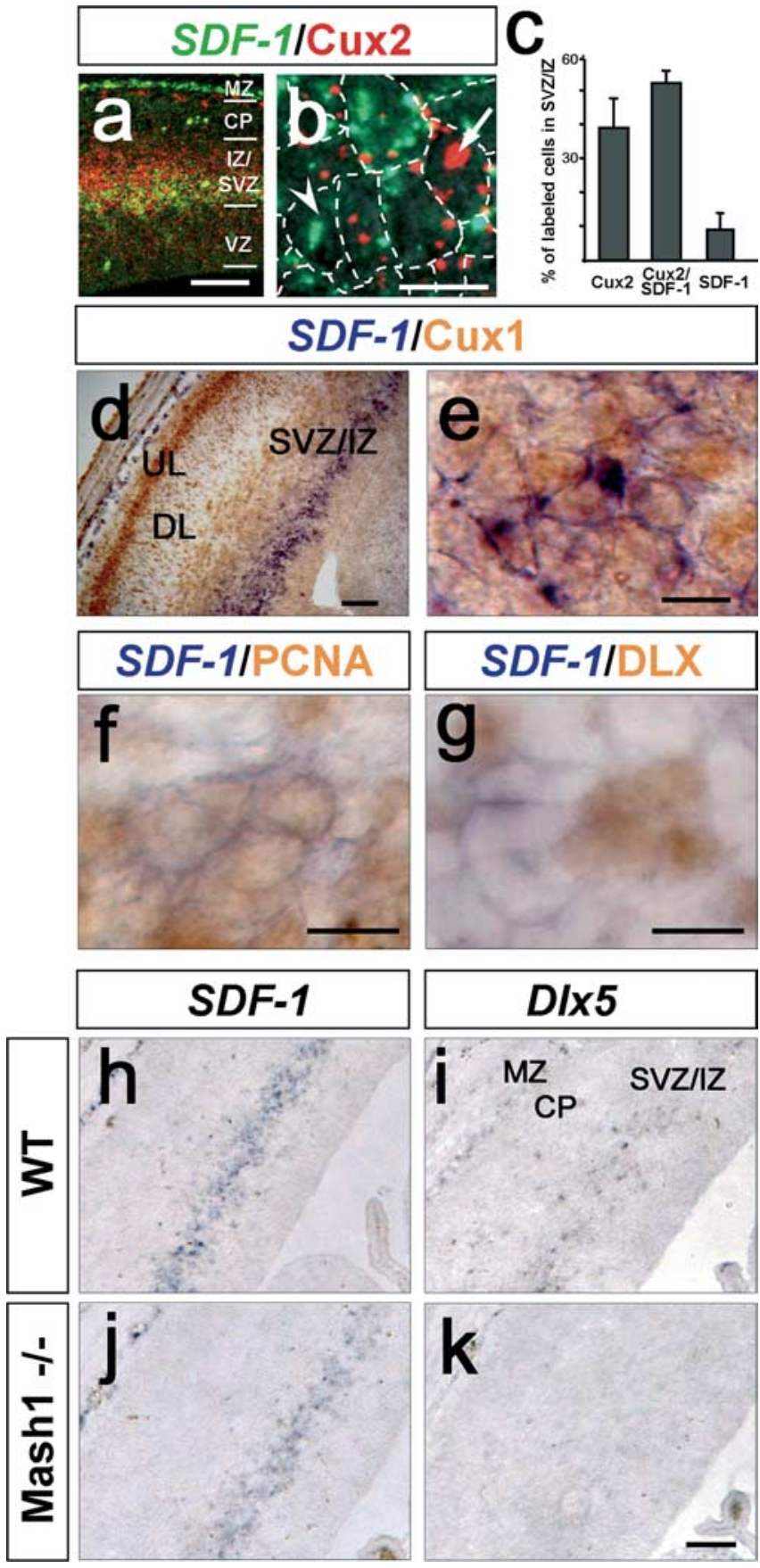

Figure 2. Identification of SDF-1-expressing cells in the SVZ/IZ. $\boldsymbol{a}, \boldsymbol{b}$, Double FISH for SDF-1 (green) and (UX2 (red) at E13.5 in the pallium. $\boldsymbol{b}$, High-magnification view of the SVZ/IZ. Most cells in the deep aspect of the SVZ/IZ are colabeled (green and red precipitates in the same cell); single-labeled cells for SDF-1 (arrowhead) and for Cux2 (arrow) can be identified. c, Quantification of single- and double-labeled cell populations in the SVZ/IZ. $\boldsymbol{d}-\boldsymbol{g}$, Combined ISH for SDF-1 (blue) with immunohistochemistry (brown staining) for Cux1 $(\boldsymbol{d}, \boldsymbol{e}), \operatorname{PCNA}(\boldsymbol{f})$, and DLX $(\boldsymbol{g})$ at E15.5. $\boldsymbol{d}$, Strong Cux1 immunoreactivity is found in upper-layer neurons of the developing $C P$, whereas weaker staining is observed in precursors in the SVZ/IZ.e, Higher magnification shows that SDF-1-expressing cells in the SVZ/IZ exhibit nuclear immunoreactivity for Cux1. $f$, The majority of SDF-1-positive cells coexpress the mitotic cell marker PCNA. $\boldsymbol{g}$, SDF-1-positive cells never express the interneuron marker DLX. $\boldsymbol{h}-\boldsymbol{k}$, ISH for SDF1 $(\boldsymbol{h}, \boldsymbol{j})$ and DIx5 $(\boldsymbol{i}, \boldsymbol{k})$ at E15.5 in wild types $(\boldsymbol{h}, \boldsymbol{i})$ and Mash 1 mutants $(\boldsymbol{j}, \boldsymbol{k})$. In the wild type, SDF-1 $(\boldsymbol{h})$ and D/x5 (i) are both expressed in the SVZ/IZ. In the Mash 1 mutant brain, D/X5 $(\boldsymbol{k})$ in the SVZ/IZ is absent, indicating the lack of interneurons. However, SDF-1 expression (j) in mutants is apparently unaltered. Scale bars: $\boldsymbol{a}, \boldsymbol{d}, \boldsymbol{h}-\boldsymbol{k}, 100 \mu \mathrm{m} ; \boldsymbol{b}, \boldsymbol{e}, \boldsymbol{f}, 10 \mu \mathrm{m}$. UL, Upper-layer neurons; DL, deep-layer neurons. 


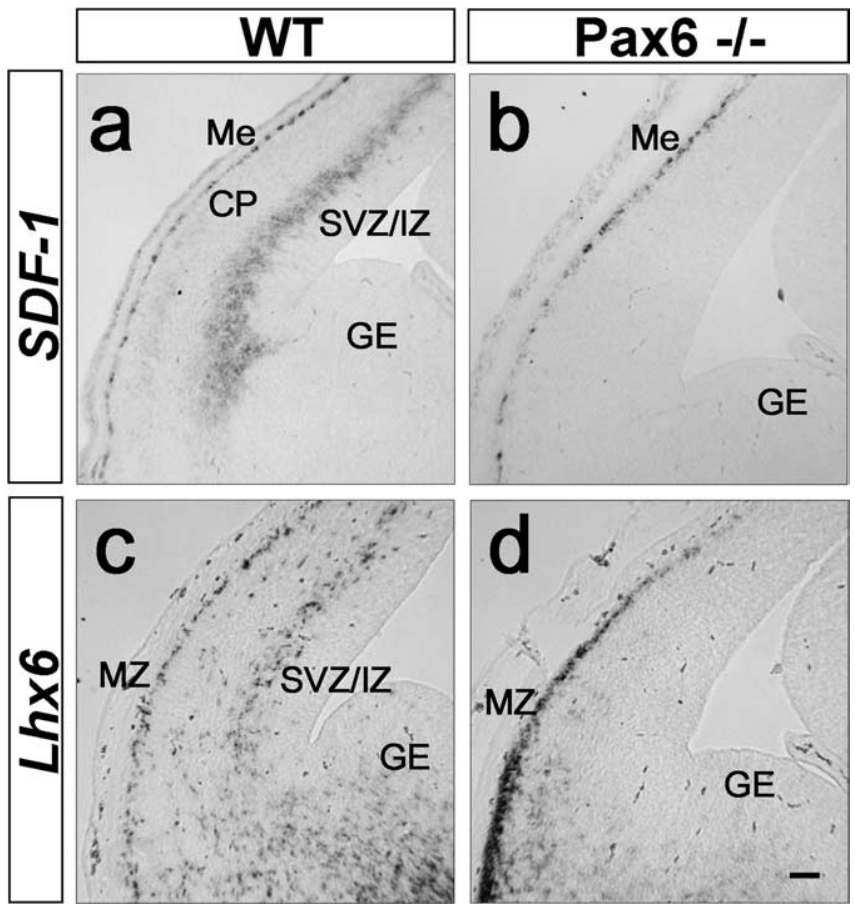

Figure 3. SDF-1 expression in the SVZ/IZ is necessary for correct interneuron migration. $\boldsymbol{a}-\boldsymbol{d}$, ISH for SDF-1 $(\boldsymbol{a}, \boldsymbol{b})$ and for the interneuron marker $\operatorname{Lhx} 6(\boldsymbol{c}, \boldsymbol{d})$ in the wild type $(\boldsymbol{a}, \boldsymbol{c})$ and in the Pax6 mutant $(\boldsymbol{b}, \boldsymbol{d})$ at E14.5. In Pax6 mutants, SDF1 mRNA expression is absent in the SVZ/IZ but remains unchanged in the meninges. At the same time, Lhx6-positive interneurons disappear from the SVZ/IZ (d), whereas larger amounts of these cells appear in the MZ pathway. Scale bar, $100 \mu \mathrm{m}$. Me, Meninges.

(2) were proliferating, and (3) were non-interneurons identified them as precursors of cortical projection neurons.

\section{SDF-1/CXCR4 signaling is essential for correct use of the SVZ/IZ pathway}

In the next step, we analyzed the functional implication of SDF-1 expression within the SVZ/IZ in interneuron migration. Pax6 mutant mice show a variety of forebrain defects including alterations in patterning, migration, and differentiation (Manuel and Price, 2005). We analyzed SDF-1 expression and interneuron migration in Pax6 mutants. For identification, we used ISH for Lhx6, a marker for pallial interneurons (Lavdas et al., 1999). ISH on brain sections of E13.5 Pax6-/- embryos showed a total absence of SDF-1 transcripts from the SVZ/IZ (Fig. 3a,b), whereas expression in the meninges was normal. In parallel, Lhx6-expressing interneurons were absent from the SVZ/IZ in Pax6 mutants but overrepresented in the MZ pathway (Fig. $3 c, d$ ). The observation that in the specific absence of SDF-1 from the SVZ/IZ more Lhx6-expressing cells were found in the MZ suggests that cells that migrate normally in the SVZ/IZ join now the $M Z$ route. This indicates a functional implication of $S D F-1$ in the routing of cortical interneurons in the SVZ/IZ.

We investigated this role of SDF-1 more directly. The SDF-1 receptor CXCR4 has been functionally implicated in neuronal cell migration (Ma et al., 1998) and shown to be expressed in the $\mathrm{MZ}$ and in individual cells within the deeper aspects of the cortex (Stumm et al., 2003). ISH at E15.5 revealed strong expression of the chemokine receptor in the MZ and in the SVZ/IZ (Fig. 4a). Double FISH analyses demonstrated that CXCR4-positive cells in the SVZ/IZ (Fig. 4b) and in the MZ (data not shown) coexpressed Lhx6, identifying them as GE-derived interneurons.

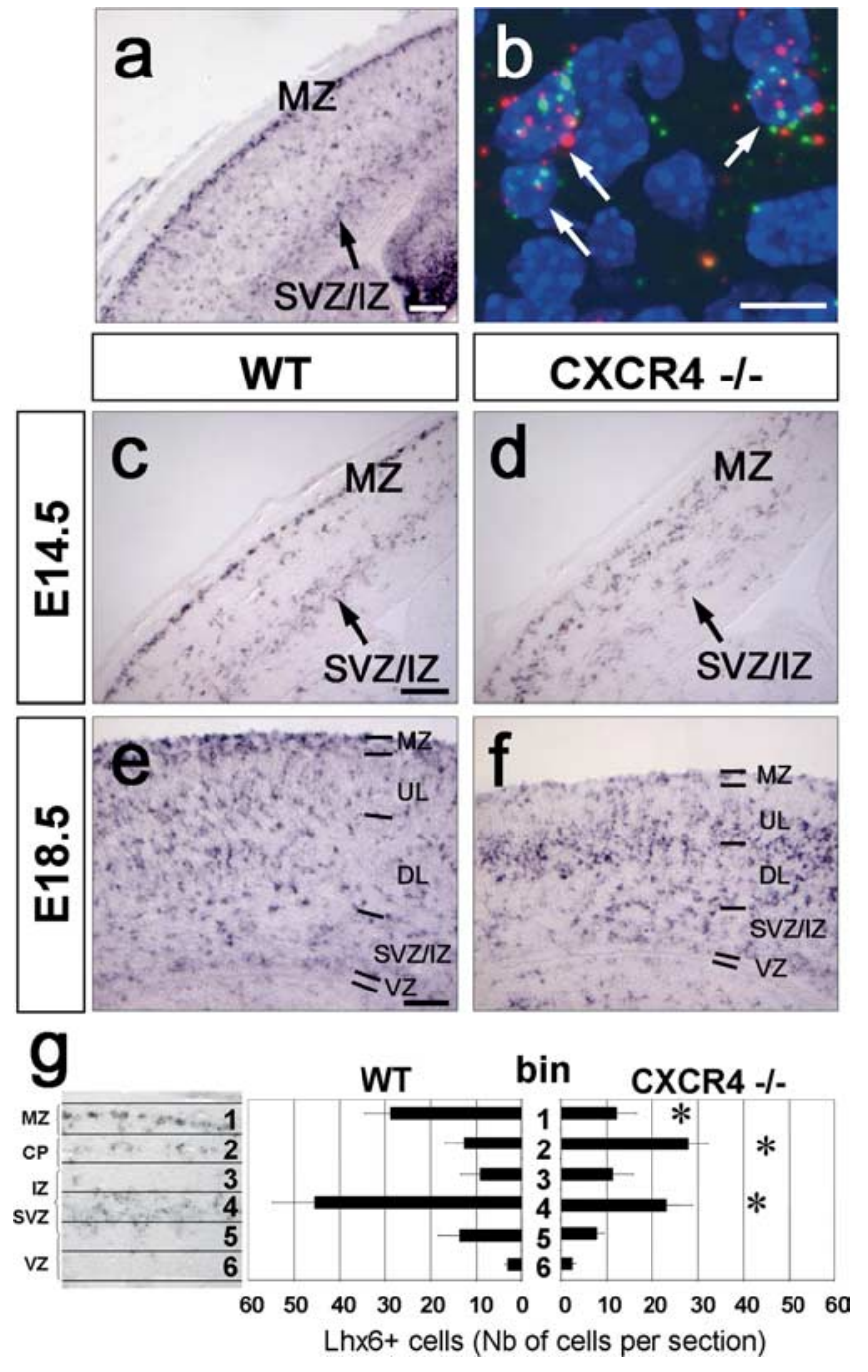

Figure 4. Altered interneuron migration in the pallium of CXCR4 mutant mice. $\boldsymbol{a}$, ISH for CXCR4 at E15.5. CXCR4 is strongly expressed in cells in the MZ and in the SVZ/IZ. $\boldsymbol{b}$, Double FISH for Lhx6 (red) and CXCR4 (green) in the SVZ/IZ. Lhx6-positive interneurons coexpress CXCR4 (arrows). $\boldsymbol{c}-\boldsymbol{f}$, ISH for Lhx6 in WT (c, e) and CXCR4 mutant $(\boldsymbol{d}, \boldsymbol{f})$ mice at E14.5 (c, $\boldsymbol{d})$ and E18.5 (e, f). $g$, Quantitative analysis of the distribution of Lhx6-expressing neurons in the pallium of WT and CXCR4 mutant E14.5 forebrain. At E14.5, invading interneurons expressing Lhx6 in the WT cortex (c) are concentrated in the SVZ/IZ and the MZ. AtE18.5, interneurons are relatively evenly distributed over the entire $(P$ in the wild type $(\boldsymbol{e})$, while in the mutants $(\boldsymbol{f})$ they accumulate in intermediate positions. Quantitative analysis showed that in $X X C R 4$ mutants (d), both SVZ/IZ and $M Z$ contain significantly less interneurons, whereas intermediate routes are preferentially used ( ${ }^{*} p>0.05$, significant differences between bins in wild type and mutant). Scale bars: $\boldsymbol{a}$, $\boldsymbol{c}-\boldsymbol{f}, 100 \mu \mathrm{m} ; \boldsymbol{b}, 10 \mu \mathrm{m}$. UL, Upper-layer neurons; DL, deep-layer neurons.

We investigated CXCR4-deficient mice to gain direct insight into the functional implication of SDF-1/CXCR4 signaling in the pallium. In the wild-type (WT) E14.5 cortex, the majority of interneurons were confined to the SVZ/IZ pathway, whereas a smaller population was localized to the MZ (Fig. 4c). In CXCR4 mutant mice, this pattern was strikingly altered. Here, the invading interneurons did not show a clear preference for the $\mathrm{MZ}$ or SVZ/IZ routes but migrated in a more dispersed manner and in intermediate regions of the pallium (Fig. $4 d$ ). Quantitative analysis of the distribution of $L h x 6$-positive cells verified these observations by showing that the MZ (Fig. $4 g$, bin 1) and the SVZ/IZ (Fig. $4 g$, bin 4) contained significantly less neurons in mutant than in WT cortex. In contrast, in mutants there was a significant increase in cell number in bin 2, representing the CP. The total 
number of cells per section was not significantly different between control and mutant animals (wild type, $114.8 \pm 21.8$; mutant, $85.9 \pm 18.7$ ).

CXCR4-deficient mice die shortly after birth. Therefore, we investigated the positioning of interneurons in the absence of the $S D F-1$ receptor at the latest possible time point, E18.5. At this age, Lhx6-stained cells in wild types were still concentrated in the MZ, whereas few cells were visible in the SVZ/IZ (Fig. 4e). Furthermore, individual Lhx6-positive cells were distributed over the entire CP. In CXCR4 mutants, the cortex was reduced in size (Fig. $4 f$ ) but showed a generally normal organization (data not shown). Here, neither the MZ nor the SVZ/IZ showed a particular accumulation of interneurons, but $L h x 6$-expressing cells were found predominantly in an intermediate position within the $\mathrm{CP}$ (Fig. 4f).

In conjunction with our observations in Pax6 mutants, these results demonstrate that SDF-1/CXCR4 signaling is functionally implicated in defining the SVZ/IZ and the MZ as immigration routes for interneurons in the pallium.

\section{Discussion}

We show here that the chemokine SDF-1 is transiently expressed by the precursors of projection neurons in the SVZ/IZ of the neocortex. This expression is essential for the correct routing of interneurons that invade the pallium from the underlying subpallium via this major pathway.

\section{SDF-1/CXCR4 signaling in interneuron migration}

During corticogenesis, $S D F-1$ has been shown to be specifically expressed in the meninges above the $\mathrm{MZ}$ as well as in a deep domain (Tham et al., 2001; Tissir et al., 2004; Daniel et al., 2005). Stumm et al. (2003) examined the effects of SDF-1 or CXCR4 knock-out on interneuron migration at a late stage of embryonic development (E18.5) and attributed the observed misrouting of CXCR4- or Reelin-expressing interneurons exclusively to the lack of chemoattraction by meningeal $S D F-1$. We identify here the deep expression domain of SDF-1 as the deep aspect of the SVZ/IZ, which represents the major entry route for interneurons in the pallium at this stage. Furthermore, we show that misrouting in CXCR4 knock-out or Pax6-deficient mice is detectable already at early stages of corticogenesis (at least by E14.5).

\section{Expression of $S D F-1$ by projection neuron precursors in the SVZ/IZ}

SDF-1 expression above the superficial pathway has been unambiguously attributed to meningeal cells (Tham et al., 2001), whereas its expression in the SVZ/IZ has so far not been positively attributed to a particular cell type. We show here that $S D F-1$ transcripts are localized in a deep domain within the SVZ/IZ. However, the SVZ/IZ is a highly heterogeneous region containing different cell populations from various origins and destined for different locations and differentiation pathways.

We considered different cellular sources for the expression of the chemokine. SDF-1 could be expressed by migratory interneurons themselves, thereby supporting their own migration into the cortex. An autocrine loop implicating SDF-1 and CXCR4 leading to actin reorganization and the promotion of cell migration has been described for endothelial progenitor cells after activation of PAR-1 (Smadja et al., 2005). However, SDF-1 is expressed by DLX-negative cells. These findings and the observation that $S D F-1$ expression is not obviously affected in Mash1 mutant mice excludes an autocrine expression of the chemokine.

In contrast, a considerable body of evidence allowed us to conclude that $S D F-1$ is expressed by the precursors of a subpopulation of cortical projection neurons during their amplification in the SVZ/IZ. First, SDF-1-expressing cells in this compartment are proliferative. Over the past years, it became clear that the SVZ/IZ is a major neurogenic compartment in which intermediate neuronal progenitors divide and supply neurons for the CP (Haubensak et al., 2004; Miyata et al., 2004; Noctor et al., 2004; Wu et al., 2005). Therefore, our finding that SDF-1-positive cells in the SVZ/IZ express the cell-cycle marker PCNA indicated an expression of the chemokine in principal neuron precursors.

Additional evidence for this scenario is provided by comparing SDF-1 expression to that of Cux1 and Cux2. Cux1 has been shown to be expressed in the VZ, in the SVZ/IZ, and very strongly in the upper layers of the CP (Nieto et al., 2004). Our analyses show colocalization of $S D F-1$ and nuclear Cux1 protein exclusively in the SVZ/IZ. In contrast, Cux2 expression is absent from the VZ but, like Cuxl, labels upper-layer progenitors in the SVZ/IZ and mature neurons of layers II-IV in the CP (Nieto et al., 2004; Zimmer et al., 2004). We found colocalization of SDF-1 and Cux2 in cells located in the SVZ/IZ.

\section{Control of interneuron migration by proliferating precursors of principal neurons: a new element for understanding corticogenesis}

The fact that, during their migration, interneurons in the cortex are in close contact with other neopallial cells, some of which are also migrating, has fostered the idea that one population might influence the behavior of the other. However, no precise evidence for a molecular cross talk between these populations has yet been presented. In the present study, we show that prospective principal neurons in the SVZ/IZ express SDF-1 at the right time and the right place to act on tangentially migrating prospective interneurons, which express the SDF-1 receptor CXCR4. We furthermore demonstrate that the laminar distribution of $\operatorname{Lh} \times 6$-positive interneurons invading from the GE is radically altered when $S D F-1$ expression in the SVZ/IZ is lacking, which is the case in Pax6 knock-out mice. Altogether, our data represent the first evidence that precursors of the two principal cell populations in the neocortex, glutamatergic projection neurons and GABAergic interneurons, are molecularly linked during their amplification/migration phase.

\section{References}

Casarosa S, Fode C, Guillemot F (1999) Mash1 regulates neurogenesis in the ventral telencephalon. Development 126:525-534.

Daniel D, Rossel M, Seki T, König N (2005) Stromal cell-derived factor-1 (SDF-1) expression in embryonic mouse cerebral cortex starts in the intermediate zone close to the pallial-subpallial boundary and extends progressively towards the cortical hem. Gene Expr Patterns 5:317-322.

Dufour HD, Chettouh Z, Deyts C, de Rosa R, Goridis C, Joly JS, Brunet JF (2006) Precraniate origin of cranial motoneurons. Proc Natl Acad Sci USA 103:8727-8732.

Favor J, Peters H, Hermann T, Schmahl W, Chatterjee B, Neuhauser-Klaus A, Sandulache R (2001) Molecular characterization of Pax6(2Neu) through Pax6(10Neu): an extension of the Pax6 allelic series and the identification of two possible hypomorph alleles in the mouse Mus musculus. Genetics 159:1689-1700.

Guillemot F, Lo LC, Johnson JE, Auerbach A, Anderson DJ, Joyner AL (1993) Mammalian achaete-scute homolog 1 is required for the early development of olfactory and autonomic neurons. Cell 75:463-476.

Haubensak W, Attardo A, Denk W, Huttner WB (2004) Neurons arise in the basal neuroepithelium of the early mammalian telencephalon: a major site of neurogenesis. Proc Natl Acad Sci USA 101:3196-3201.

Lavdas AA, Grigoriou M, Pachnis V, Parnavelas JG (1999) The medial ganglionic eminence gives rise to a population of early neurons in the developing cerebral cortex. J Neurosci 19:7881-7888. 
Ma Q, Jones D, Borghesani PR, Segal RA, Nagasawa T, Kishimoto T, Bronson RT, Springer TA (1998) Impaired B-lymphopoiesis, myelopoiesis, and derailed cerebellar neuron migration in CXCR4- and SDF-1-deficient mice. Proc Natl Acad Sci USA 95:9448-9453.

Manuel M, Price DJ (2005) Role of Pax6 in forebrain regionalization. Brain Res Bull 66:387-393.

Marin O, Rubenstein JL (2003) Cell migration in the forebrain. Annu Rev Neurosci 26:441-483.

Metin C, Baudoin JP, Rakic S, Parnavelas JG (2006) Cell and molecular mechanisms involved in the migration of cortical interneurons. Eur J Neurosci 23:894-900.

Miyata T, Kawaguchi A, Saito K, Kawano M, Muto T, Ogawa M (2004) Asymmetric production of surface-dividing and non-surface-dividing cortical progenitor cells. Development 131:3133-3145.

Nieto M, Monuki ES, Tang H, Imitola J, Haubst N, Khoury SJ, Cunningham J, Gotz M, Walsh CA (2004) Expression of Cux-1 and Cux-2 in the subventricular zone and upper layers II-IV of the cerebral cortex. J Comp Neurol 479:168-180.

Noctor SC, Martinez-Cerdeno V, Ivic L, Kriegstein AR (2004) Cortical neurons arise in symmetric and asymmetric division zones and migrate through specific phases. Nat Neurosci 7:136-144.

Panganiban G, Sebring A, Nagy L, Carroll S (1995) The development of crustacean limbs and the evolution of arthropods. Science 270:1363-1366.

Rash BG, Grove EA (2006) Area and layer patterning in the developing cerebral cortex. Curr Opin Neurobiol 16:25-34.

Smadja DM, Bieche I, Uzan G, Bompais H, Muller L, Boisson-Vidal C, Vidaud M, Aiach M, Gaussem P (2005) PAR-1 activation on human late endothelial progenitor cells enhances angiogenesis in vitro with upregu- lation of the SDF-1/CXCR4 system. Arterioscler Thromb Vasc Biol 25:2321-2327.

Stumm RK, Zhou C, Ara T, Lazarini F, Dubois-Dalcq M, Nagasawa T, Hollt V, Schulz S (2003) CXCR4 regulates interneuron migration in the developing neocortex. J Neurosci 23:5123-5130.

Takahashi T, Caviness Jr VS (1993) PCNA-binding to DNA at the G1/S transition in proliferating cells of the developing cerebral wall. J Neurocytol 22:1096-1102.

Tham TN, Lazarini F, Franceschini IA, Lachapelle F, Amara A, Dubois-Dalcq M (2001) Developmental pattern of expression of the alpha chemokine stromal cell-derived factor 1 in the rat central nervous system. Eur J Neurosci 13:845-856.

Tissir F, Wang CE, Goffinet AM (2004) Expression of the chemokine receptor Cxcr4 mRNA during mouse brain development. Brain Res Dev Brain Res 149:63-71.

Tiveron MC, Hirsch MR, Brunet JF (1996) The expression pattern of the transcription factor Phox2 delineates synaptic pathways of the autonomic nervous system. J Neurosci 16:7649-7660.

Wu SX, Goebbels S, Nakamura K, Nakamura K, Kometani K, Minato N, Kaneko T, Nave KA, Tamamaki N (2005) Pyramidal neurons of upper cortical layers generated by NEX-positive progenitor cells in the subventricular zone. Proc Natl Acad Sci USA 102:17172-17177.

Zimmer C, Tiveron MC, Bodmer R, Cremer H (2004) Dynamics of Cux2 expression suggests that an early pool of SVZ precursors is fated to become upper cortical layer neurons. Cereb Cortex 14:1408-1420.

Zou YR, Kottmann AH, Kuroda M, Taniuchi I, Littman DR (1998) Function of the chemokine receptor CXCR4 in haematopoiesis and in cerebellar development. Nature 393:595-599. 